\title{
A cellular automata traffic flow model considering the heterogeneity of acceleration and delay probability
}

\author{
Qi-Lang Li ${ }^{\mathrm{a}, \mathrm{b}, *}$, S. C. Wong ${ }^{\mathrm{b}}$, Jie Min ${ }^{\mathrm{a}}$, Shuo Tian ${ }^{\mathrm{c}}$, Bing-Hong Wang ${ }^{\mathrm{d}}$ \\ ${ }^{a}$ School of Mathematics and Physics, Anhui Jianzhu University, Hefei 230601, China \\ ${ }^{b}$ Department of Civil Engineering, The University of Hong Kong, Hong Kong, China \\ ${ }^{c}$ School of Social Development and Public Policy, Fudan University, Shanghai 200433, \\ China \\ ${ }^{d}$ Department of Modern Physics, University of Science and Technology of China, Hefei \\ 230026, China
}

\begin{abstract}
This study examines the cellular automata traffic flow model, which considers the heterogeneity of vehicle acceleration and the delay probability of vehicles. Computer simulations are used to identify three typical phases in the model: free-flow, synchronized flow, and wide moving traffic jam. In the synchronized flow region of the fundamental diagram, the low and high velocity vehicles compete with each other and play an important role in the evolution of the system. The analysis shows that there are two types of bistable phases. However, in the original Nagel and Schreckenberg cellular automata traffic model, there are only two kinds of traffic conditions, namely, free-flow and traffic jams. The synchronized flow phase and bistable phase have not been found.

Keywords: Traffic flow, cellular automata, heterogeneity, acceleration, delay probability
\end{abstract}

\section{Introduction}

Traffic science examines the fundamental laws and properties of transportation systems. The traffic dynamic behavior of vehicular traffic is complex and

\footnotetext{
* Corresponding author

Email addresses: qilang@mail.ustc.edu.cn (Qi-Lang Li), hhecwsc@hku.hk (S. C. Wong), minjie@ahjzu.edu.cn (Jie Min), bhwang@ustc.edu.cn (Bing-Hong Wang)
} 
has diverse, interesting, non-equilibrium features such as collective behavior, self-organization, coexisting phases, etc.[1, 2, 3, 4, 5, 6, 7].

Freeway traffic flow is a very complex spatiotemporal nonlinear dynamic process [1]. For this reason, empirical traffic pattern features are not fully understood. The three-phase traffic flow theory can explain empirical spatiotemporal traffic patterns better than earlier traffic flow theories $[8,9,10]$. Previous experimental studies have shown that the complexity in traffic flow is linked to diverse space-time transitions between three basically different kinds of traffic: free traffic flow, synchronized traffic flow, and traffic jams [11]. Many existing cellular automata traffic flow models are based on Kerner's three-phase traffic flow theory $[12,13,14,15,16,17,18,19,20,21,22,23,24,25,26,27,28,29]$.

The cellular automata (CA) model is useful for simulating large systems and a variety of CA models have been proposed [3, 30, 31, 32, 33, 29, 34, 35, 36, 37]. Nagel and Schreckenberg (NS) [36] proposed a model of gradually increasing velocity, in which a vehicle may increase by only one unit per time step. In the Fukui-Ishibashi (FI) model [37], vehicles can move only $m$ sites in one time step, and only if they are not blocked by vehicles in front. The notation $m$, which denotes the maximum velocity of vehicles, must be a positive integer. It is evident that these two popular one-dimensional traffic flow models are based on different vehicle accelerations. In real traffic, vehicle acceleration depends on their own current velocity, vehicle performance, driving habits, and so on; therefore, real acceleration must be a random number somewhere between 0 and the maximum velocity of vehicles.

The randomization of update rules of the NS and FI models [36, 37] can provide the key to modeling the formation of spontaneously emerging traffic jams and natural speed fluctuations caused by human behavior or varying external conditions. In the NS model, if the velocities of vehicles are greater than zero, all vehicles have the same delay probability [36]. The FI model [37] has similar results if the velocities are equal to 5 . However, Brilon and $\mathrm{Wu}$ argued that this rule has no theoretical basis and is in fact heuristic [38]. Randomization occurs inevitably in actual traffic and it is evident that different drivers have different 
delay probabilities under various velocities, external conditions, etc.

The heterogeneity of vehicular traffic is an important feature in traffic flow studies. Different types of driver's individual property and/or vehicles (e.g., vehicles with different maximal velocity, length, and so on) have been considered in some studies $[39,40,41,42,43,44,45,46,47,48,49,50,51,52,53,49]$. Unlike these studies, this study establishes a new cellular automata traffic flow model that considers the heterogeneity in the acceleration and delay probability of vehicles. Our model can reproduce some common characteristics of the real traffic, such as the start-and-stop waves, and present the synchronized flow phase and bistable phase. The fundamental diagram of the model shows quantitative coincidence of maximum flow with values taken from real traffic measurements.

\section{Definition of the CA traffic model}

For the sake of completeness, we now briefly define the NS model. In the NS model, a road is composed of $L$ cells of equal size, and every single cell can be empty or occupied by a single vehicle. The velocity of each vehicle can be one of the $v_{\max }+1$ allowed integer values, i.e., $v=0,1, \ldots, v_{\max }$. Here, $v_{\max }$ (the notation $v_{\max }$ is a positive integer) denotes the maximum velocities of vehicles. Let $v_{\max }$ be 5 in this study. Below, the periodic boundary conditions are adopted and no vehicle is allowed to overtake on the road.

As cellular automata are dynamic systems that are discrete in nature, the acceleration of each vehicle must be a random integer. However, the acceleration values of vehicles can be no larger than their maximum velocities. Thus, the acceleration of each vehicle is also one of the $v_{\max }+1$ allowed integer values, $v=0,1, \ldots, v_{\max }$.

As mentioned above, we only consider the effect of velocity on the delay probability of vehicles. The delay probability of each vehicle exists when the value of its possible velocity equals the value of its corresponding gap, and the probability varies with the size of the corresponding gap. We assume that the delay probability of each vehicle increases linearly with its velocity. As Table 1 
shows, $p(v)=0$ where $v(t)=1$. In other words, the vehicle does not need to slow down if $v(t)=g a p=1$, which is similar to the conditions in [30].

The state of the road at time $t+1$ can be obtained from time step $t$ by simultaneously applying the following rules to all vehicles (parallel dynamics): (i) the randomization parameter of acceleration $a$ is determined by $a=$ $\operatorname{rand}() /\left(v_{\max }+1\right)$, where $\operatorname{rand}()$ function is a random function that can generate random numbers. (ii) acceleration, velocity is $v_{i}(t+1) \rightarrow \min \left(v_{i}(t)+a, V_{\max }\right)$; (iii) deceleration due to other vehicles, velocity is $v_{i}(t+1) \rightarrow \min \left(v_{i}(t+1), g a p\right)$, where gap is the number of empty cells in front of the $i$ th vehicle; (iv) the parameter of the delay probability is determined by $p=p(v) ;(\mathrm{v})$ randomization with probability $p$ if $v_{i}(t+1)=g a p, v_{i}(t+1) \rightarrow \max \left(v_{i}(t+1)-1,0\right)$; and (vi) vehicle movement is $x_{i}(t+1) \rightarrow x_{i}(t)+v_{i}(t+1)$, where $v_{i}(t)$ and $x_{i}(t)$ are the velocity and the position of the $i$ th vehicle at the current $t$ time step, respectively.

In computer simulations, the length of each cell is set to $7.5 \mathrm{~m}$. System size $L$ is assumed to be 2000, which corresponds to an actual road length of around $15 \mathrm{~km}$. One time step approximately corresponds to $1 \mathrm{~s}$ in real time. Thus, the maximum velocity $v_{\max }=5$ corresponds to $135 \mathrm{~km} / \mathrm{h}$ in real traffic.

Table 1: Delay probability depends on the velocities of the vehicles

\begin{tabular}{|c|c|c|c|c|c|c|c|}
\hline$v(t)=$ gap & 1 & 2 & 3 & 4 & 5 & $\cdots$ & Linear function expression \\
\hline$p(v)$ & 0 & 0.1 & 0.2 & 0.3 & 0.4 & $\cdots$ & $p(v)=[v(t)-1] / 2 v_{\max }$ \\
\hline
\end{tabular}

\section{Rich behavior of the present model}

The fundamental diagrams (FD) of the present model and the NS model are presented in Figure 1(a). There are only two kinds of traffic conditions, namely, free- flow and traffic jam in the NS model [33]. However, in the present model, there are three phases: (i) free-flow, (ii) synchronized flow, and (iii) wide moving jam. Moreover, from Figure 1(a), the maximum flow of the present model is 

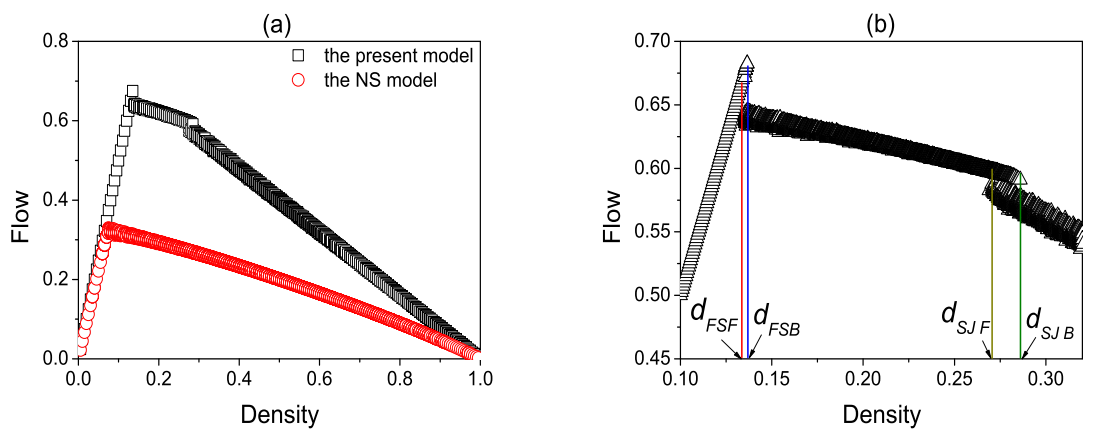

Figure 1: (a) Fundamental diagram (FD) of the present model and the NS model. (b) Magnified sections of the FD of the present model in Fig. 1(a)

0.675 , which is closer to the measurement value of 0.69 , in comparison with 0.32 of the NS model. Compared with the NS model, the system of the present model reaches the maximum flow under a higher density, and then this system still maintains a higher flow within a wider density range. Based on these results, the transit capacity of the present model is more realistic which is superior to the NS model. In the following sections, we will discuss the specific feature of three phases for the present model.

In the free-flow region $\left(0<d<d_{F S F}\right)$, shown in Fig. 1(b), the delay probability does not work for all vehicles. Vehicles in the system move at the maximum velocity because they can self-organize, and gap $>v_{\max }$ holds for all vehicles. Let us look at the structure of the free flow in the present model. As shown in Fig. 2(a), the trajectory of each vehicle must be a straight line (red line) in the time and space plots.

The jamming region $\left(d_{S J B}<d<1\right)$ in Fig. 1(b), presented in detail in Fig. 2(b), shows the typical spatiotemporal structure of a wide moving jam (the black band). This black band grows wider as traffic density increases and finally becomes a completely compact jam when density $d=1$. In the black band internal, the wide moving jam is completely compact and the density of vehicles is 1 . All of the vehicles are at a standstill, bumper to bumper, until the 
(a)

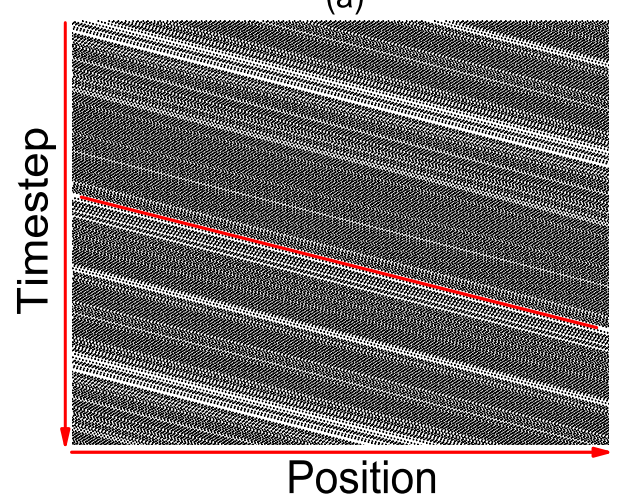

(b)

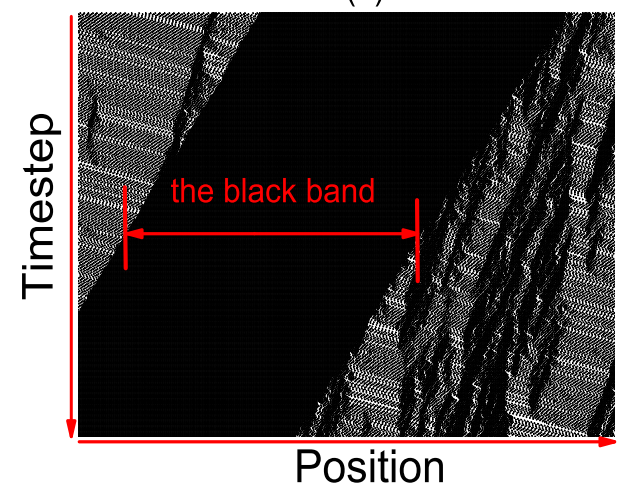

Figure 2: The space-time plots of the present model, where (a) $d=0.12$ and (b) $d=0.40$. The vehicles are moving from the left to the right, and the vertical direction (down) is (increasing) time, as shown by the two black arrows. 
(a)

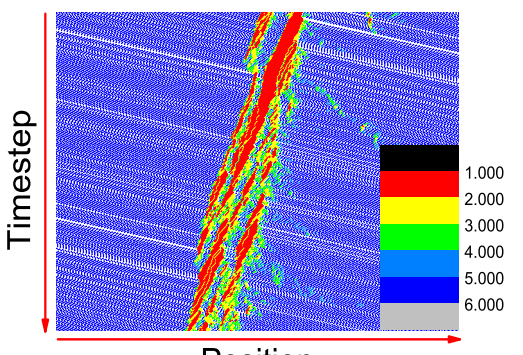

Position (b)

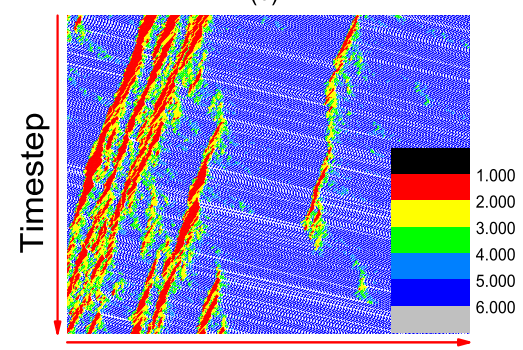

Position

(c)

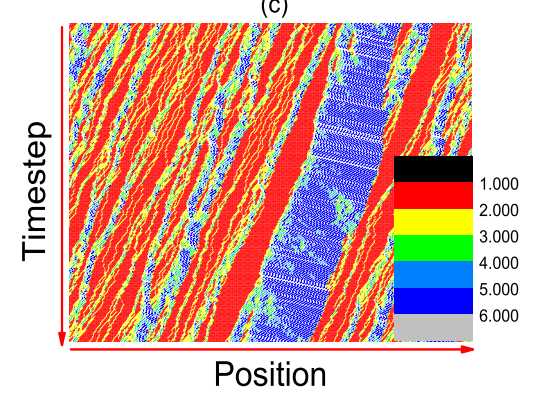

Figure 3: Evolution of the spatiotemporal pattern of vehicle velocity in the simulation of synchronized flow where (a) $d=0.15$, (b) $d=0.20$, and (c) $d=0.25$.

downstream front of the jam moves forward.

The synchronized flow region $\left(d_{F S B}<d<d_{S J F}\right)$ of Fig. 1(b), presented in detail in Fig. 3, shows the typical spatiotemporal distribution plots of velocity. Black pixel points indicate that the vehicles have velocities equal to zero. There are no black pixel points in Fig. 3, indicating that all of these vehicles are moving forward. Vehicles with the same velocity gather together and form many small teams. These features reflect the characteristics of a synchronous flow. Moreover, Fig. 3 shows that with an increase in density, there are fewer and fewer blue pixel points (a blue pixel point indicates a vehicles with a velocity equal to 5), and more red pixel points (a red pixel point indicates a vehicle with a velocity equal to 1). The red pixel points gather together and form many small teams. There are similar examples of other color pixel points such as yellow, 

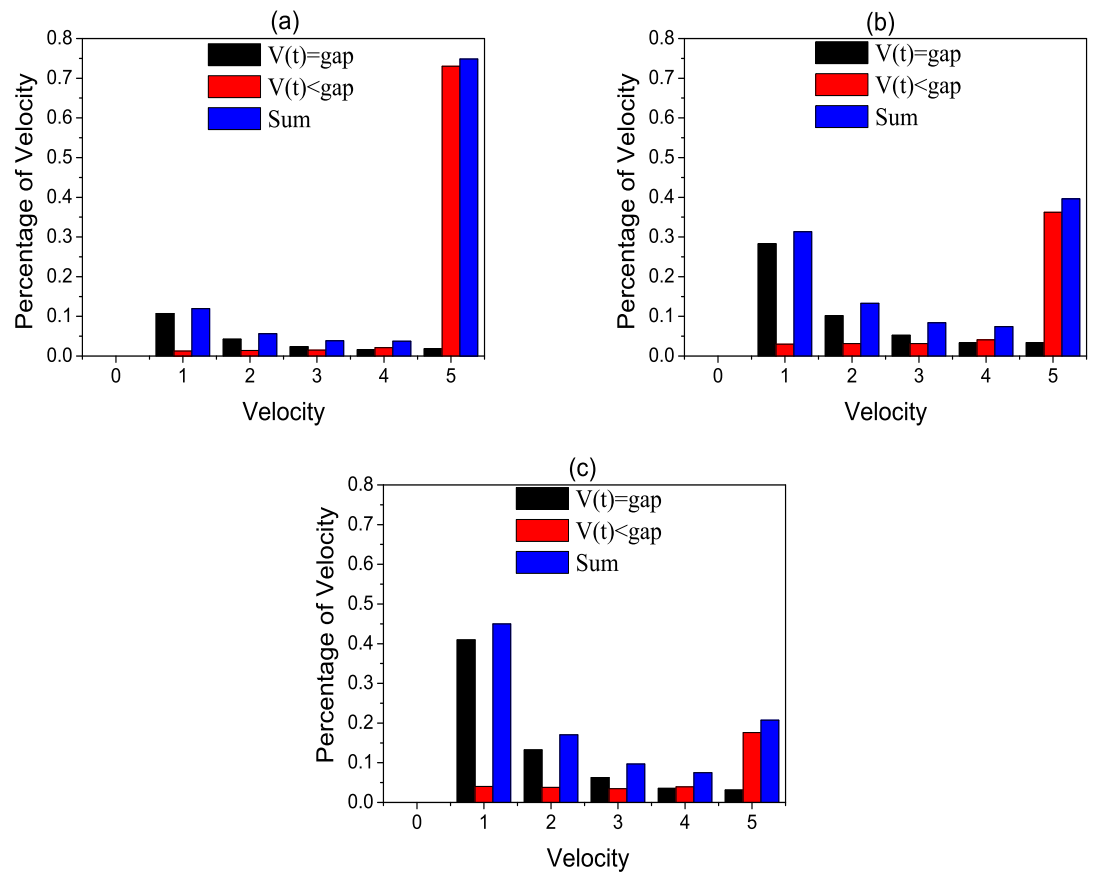

Figure 4: Statistical distribution plots of velocity for synchronized flow where (a) $d=0.15$, (b) $d=0.20$, and (c) $d=0.25$. Black bars denote the case where $v(t)=$ gap. Red bars denote the case where $v(t)<$ gap. Blue bars denote the case where the sum of $v(t)=$ gap and $v(t)<$ gap.

green, and so on, because the average temporal headway (gap) shortens with an increase in density, leading to more low-velocity vehicles.

Fig. 3 only presents one sample with a given density, and other cases are not discussed. Fig. 4 shows the statistical distributions of velocity; and all of the values are an average of 50 samples. As in Fig. 4, there are no vehicles with a velocity equal to zero. The blue bars in the histograms indicate that the percentage of vehicles in the system with velocity 5 decreases quickly from $75 \%$ to $21 \%$ with increasing density, as shown in Fig. 4. However, the percentage of vehicles traveling at other velocities increases with density. The percentage of vehicles traveling at velocity 1 increases from $12 \%$ to $45 \%$. No matter whether density is high or low, vehicles with velocities 1 and 5 always have a strong effect 


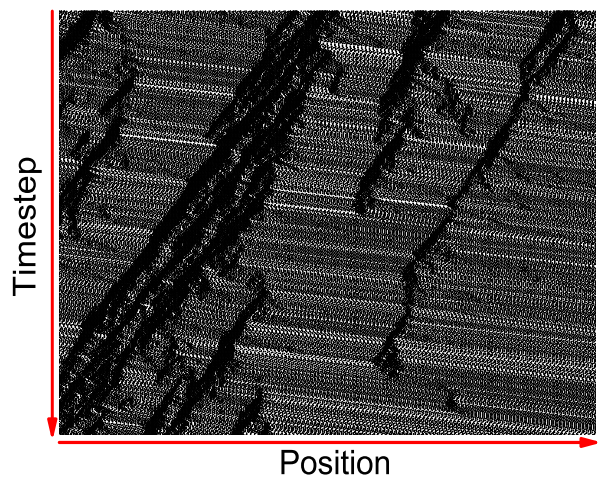

Figure 5: The space-time plot of synchronized flow in the present model, where $d=0.20$. The vehicles are moving from the left to the right, and the vertical direction (down) is (increasing) time as shown by the two black arrows.

on synchronized flow. Moreover, for $v(t)=1,2$, or 3 , the number of vehicles for the case of $v(t)=g a p$ is greater than for $v(t)<$ gap. However, this is reversed for $v(t)=4$ or 5 . Furthermore, there is a typical "stop-and-go" phenomenon, as shown in Fig. 5, which is obviously induced by vehicles with velocities equal to 1.

Fig. 6 shows that there are two different types of bistable phases: FS and SJ. For the bistable state phase FS $\left(d_{F S F} \leq d \leq d_{F S B}\right)$, after a sufficiently long period of time, the system evolves into a free flow under some initial configurations, but under other initial configurations, the system evolves into a synchronized flow. In the bistable state phase SJ $\left(d_{S J F} \leq d \leq d_{S J B}\right)$, the system evolves into a synchronized flow under some initial configurations, but under other initial configurations, the system evolves into a wide moving jam. Changes in system size do not affect the bistability. 

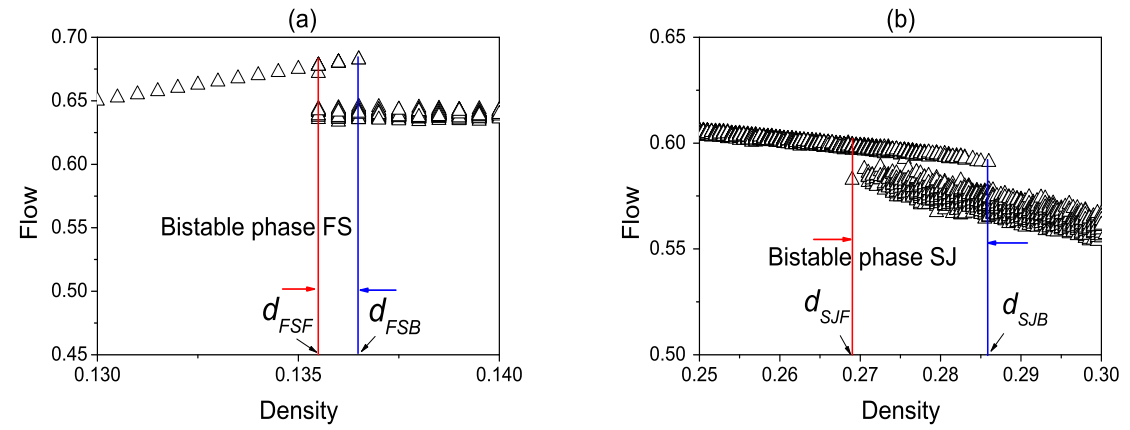

Figure 6: (a) Magnified sections near $d_{F S F}$ and $d_{F S B}$ given in Fig. 1(b), (b) Magnified sections near $d_{S J F}$ and $d_{S J B}$ given in Fig. 1(b).

(a)

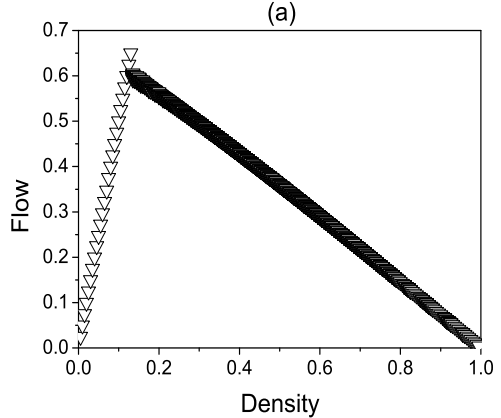

(b)

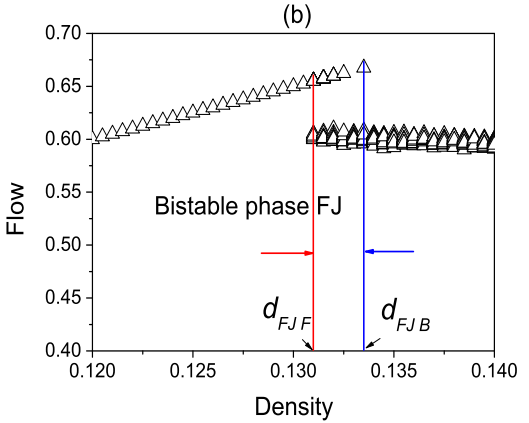

Figure 7: (a) Fundamental diagram of the present model in Table 2. (b) Magnified sections of the FD given in Fig. 7(a) 
Table 2: Values of the delay probability depend on the velocities of vehicles

\begin{tabular}{|c|c|c|c|c|c|c|c|}
\hline$v(t)=$ gap & 1 & 2 & 3 & 4 & 5 & $\cdots$ & Linear function expression \\
\hline$p(v)$ & 0.1 & 0.2 & 0.3 & 0.4 & 0.5 & $\cdots$ & $p(v)=v(t) / 2 v_{\max }$ \\
\hline
\end{tabular}

\section{Conclusions and Discussions}

To summarize, this study examines the heterogeneity of acceleration and delay probability of vehicles in the cellular automata traffic flow model. For all vehicles, if their velocity is the same as the velocity of the corresponding gaps, a random delay is possible, and the value of the delay probability increases linearly with velocity according to the equation $p(v)=[v(t)-1] / 2 v_{\max }$. The acceleration of each vehicle is a random integer between zero and the maximum velocity. The computer simulations identify three typical phases in the fundamental diagram of the present model: free flow, synchronized flow, and wide moving jam. In the synchronized flow region, vehicles with low and high velocities play an important role. As density increases, the number of low velocity vehicles increases, and the number of high velocity vehicles decreases. Furthermore, there are two types of bistable phases depending on the initial distribution of the vehicles. The system eventually evolves into one of two configurations after a sufficient period of time.

Moreover, as Table 2 shows, if we set $p(v)=0.1$, where $v(t)=1$, then, as shown in Fig. 7(a), there are only two typical phases: free flow and traffic jams. It is evident that the delay probability does not exist for vehicles with a velocity of 1 and this is the key factor in the synchronized flow region in the FD of the present model. Moreover, as shown in Fig. 7(b), we have identified a new type of bistable phase, FJ. Under some initial configurations, the system will evolve into free flow ; In other initial configurations, the system will evolve into a traffic jam.

In real traffic, the delay probability of each vehicle is influenced by many factors such as driver's individual aspects, vehicle type, etc. This study only considers the delay probability of each vehicle that depends on its velocity in 
the linear functional form. In our future work, the delay probability of vehicles with nonlinear functional form, and the consideration of other relevant factors will be further investigated.

\section{Acknowledgments}

QL Li is supported by the Natural Science Foundation of China under Grant No. 11302001, the Anhui Provincial Natural Science Foundation under Grant No. 1308085QA11, and the Key Project of Support Plan for the Outstanding Youth of Anhui Provincial Colleges and Universities No. gxyqZD2016145. SC Wong is supported by the University Research Committee of the University of Hong Kong (201411159005). J Min is supported by the Natural Science Foundation of China under Grant No. 71571002. BH Wang is supported by the Natural Science Foundation of China under Grant No. 11275186.

[1] B. S. Kerner, The physics of traffic: empirical freeway pattern features, engineering applications, and theory, Springer, 2012.

[2] T. Nagatani, Reports on Progress in Physics 65 (9) (2002) 1331.

[3] S. Maerivoet, B. De Moor, Physics Reports 419 (1) (2005) 1-64.

[4] D. Chowdhury, L. Santen, A. Schadschneider, Physics Reports 329 (4) (2000) 199-329.

[5] Q.-L. Li, R. Jiang, B.-H. Wang, M.-R. Liu, EPL 99 (3) (2012) 38004.

[6] S. Boccaletti, G. Bianconi, R. Criado, C. I. Del Genio, J. Gómez-Gardeñes, M. Romance, I. Sendina-Nadal, Z. Wang, M. Zanin, Physics Reports 544 (1) (2014) 1-122.

[7] J. Tanimoto, T. Fujiki, Z. Wang, A. Hagishima, N. Ikegaya, Journal of Statistical Mechanics: Theory and Experiment 2014 (11) (2014) P11027.

[8] B. S. Kerner, S. L. Klenov, G. Hermanns, P. Hemmerle, H. Rehborn, M. Schreckenberg, Physical Review E 88 (5) (2013) 054801. 
[9] B. S. Kerner, S. L. Klenov, M. Schreckenberg, Journal of Statistical Mechanics: Theory and Experiment 2014 (3) (2014) P03001.

[10] B. S. Kerner, P. Hemmerle, M. Koller, G. Hermanns, S. L. Klenov, H. Rehborn, M. Schreckenberg, Physical Review E 90 (3) (2014) 032810.

[11] B. S. Kerner, H. Rehborn, Physical Review E 53 (5) (1996) R4275.

[12] B. S. Kerner, S. L. Klenov, D. E. Wolf, Journal of Physics A: Mathematical and General 35 (47) (2002) 9971.

[13] B. S. Kerner, S. L. Klenov, Journal of Physics A: Mathematical and General 35 (3) (2002) L31.

[14] B. S. Kerner, S. L. Klenov, M. Schreckenberg, Physical Review E 84 (4) (2011) 046110.

[15] B. S. Kerner, Physical Review E 85 (3) (2012) 036110.

[16] B. S. Kerner, S. L. Klenov, G. Hermanns, M. Schreckenberg, Physica A: Statistical Mechanics and its Applications 392 (18) (2013) 4083-4105.

[17] B. S. Kerner, S. L. Klenov, M. Schreckenberg, Physical Review E 89 (5) (2014) 052807.

[18] R. Jiang, Q.-S. Wu, Journal of Physics A: Mathematical and General 37 (34) (2004) 8197.

[19] R. Jiang, Q. S. Wu, The European Physical Journal B-Condensed Matter and Complex Systems 46 (4) (2005) 581-584.

[20] J.-F. Tian, B. Jia, X.-G. Li, R. Jiang, X.-M. Zhao, Z.-Y. Gao, Physica A: Statistical Mechanics and its Applications 388 (23) (2009) 4827-4837.

[21] J.-F. Tian, Z.-Z. Yuan, B. Jia, H.-Q. Fan, T. Wang, Physics Letters A 376 (44) (2012) 2781-2787.

[22] J.-F. Tian, Z.-Z. Yuan, M. Treiber, B. Jia, W.-Y. Zhang, Physica A: Statistical Mechanics and its Applications 391 (11) (2012) 3129-3139. 
[23] J.-F. Tian, N. Jia, N. Zhu, B. Jia, Z.-Z. Yuan, Transportation Research Part C: Emerging Technologies 44 (2014) 282-298.

[24] B. Jia, X.-G. Li, T. Chen, R. Jiang, Z.-Y. Gao, Transportmetrica 7 (2) (2011) 127-140.

[25] Y. Xue, L.-S. Jia, W.-Z. Teng, W.-Z. Lu, Communications in Nonlinear Science and Numerical Simulation 22 (1) (2015) 285-296.

[26] L.-S. Jia, W.-Z. Teng, W. Pan, Y. Xue, et al., Nonlinear Dynamics 78 (3) (2014) 1801-1809.

[27] J. J. Wu, H. J. Sun, Z. Y. Gao, Physical Review E 78 (3) (2008) 036103.

[28] W. Knospe, L. Santen, A. Schadschneider, M. Schreckenberg, Journal of Physics A: Mathematical and general 33 (48) (2000) L477.

[29] R. Jiang, Q.-S. Wu, Journal of Physics A: Mathematical and General 36 (2) (2003) 381.

[30] Q.-L. Li, B.-H. Wang, M.-R. Liu, Physica A: Statistical Mechanics and its Applications 390 (7) (2011) 1356-1362.

[31] Y. Xue, L.-Y. Dong, L. Li, S.-Q. Dai, Physical Review E 71 (2) (2005) 026123.

[32] H. Kuang, G.-X. Zhang, X.-L. Li, S.-M. Lo, Physics Letters A 378 (21) (2014) 1455-1460.

[33] Z.-J. Ding, R. Jiang, Z.-Y. Gao, B.-H. Wang, J. Long, Physical Review E 88 (2) (2013) 022809.

[34] B. Jia, R. Jiang, Q.-S. Wu, Physical Review E 69 (5) (2004) 056105.

[35] J.-X. Ding, H.-J. Huang, Q. Tian,Chinese Physics B 20 (2) (2011) 028901.

[36] K. Nagel, M. Schreckenberg, Journal de Physique I 2 (12) (1992) 2221-2229. 
[37] M. Fukui, Y. Ishibashi, Journal of the Physical Society of Japan 65 (6) (1996) 1868-1870.

[38] W. Brilon, N. Wu, Evaluation of cellular automata for traffic flow simulation on freeway and urban streets, in: traffic and mobility: simulationeconomics-environment, Springer Berlin Heidelberg, 1999.

[39] H. Ez-Zahraouy, K. Jetto, A. Benyoussef, The European Physical Journal B-Condensed Matter and Complex Systems 40 (1) (2004) 111-117.

[40] N. Lakouari, K. Bentaleb, H. Ez-Zahraouy, A. Benyoussef, Physica A: Statistical Mechanics and its Applications 439 (2015) 132-141.

[41] B. S. Kerner, S. L. Klenov, Journal of Physics A: Mathematical and General 37 (37) (2004) 8753.

[42] S. C. Wong, H. Yang, W. S. A. Yeung, S. L. Cheuk, M. K. Lo, Journal of Transportation Engineering 124 (3) (1998) 229-234.

[43] Q.-L. Li, R. Jiang, J. Min, J.-R. Xie, B.-H. Wang, EPL 108 (2) (2014) 28001.

[44] Q.-L. Li, B.-H. Wang, M.-R. Liu, Physica A: Statistical Mechanics and its Applications 389 (21) (2010) 5045-5052.

[45] N. Moussa, The European Physical Journal B-Condensed Matter and Complex Systems 41 (3) (2004) 421-431.

[46] Y.-M. Yuan, R. Jiang, M.-B. Hu, Q.-S. Wu, R. Wang, Physica A: Statistical Mechanics and its Applications 388 (12) (2009) 2483-2491.

[47] Y. Luo, B. Jia, J. Liu, W. H. K. Lam, X. Li, Z. Gao, Journal of Advanced Transportation 49 (1) (2015) 29-47.

[48] T.-Q. Tang, L. Chen, R.-Y. Guo, H.-Y. Shang, Physica A: Statistical Mechanics and its Applications 440 (2015) 49-56. 
[49] T.-Q. Tang, K.-W. Xu, S.-C. Yang, H.-Y. Shang, Measurement 59 (2015) $30-37$.

[50] T.-Q. Tang, H.-J. Huang, S.-G. Zhao, H.-Y. Shang, Physics Letters A 373 (29) (2009) 2461-2466.

[51] T.-Q. Tang, C.-Y. Li, H.-J. Huang, H.-Y. Shang, Nonlinear Dynamics 67 (3) (2012) 2255-2265.

[52] T.-Q. Tang, Y.-P. Wang, G.-Z. Yu, H.-J. Huang, Communications in Theoretical Physics 58 (4) (2012) 583.

[53] T.-Q. Tang, J. He, S.-C. Yang, H.-Y. Shang, Physica A: Statistical Mechanics and Its Applications 413 (2014) 583-591. 